\title{
A Case of Euglycemic Diabetic Ketoacidosis due to Canagliflozin Complicated by Takotsubo Cardiomyopathy
}

\author{
Muzammil Khan", Shaza Khalid, Asghar Marwat, Hassan Mehmood \\ Department of Internal Medicine, Conemaugh Memorial Medical Center/ Temple University, 1086 Franklin Street, Johnstown, PA, \\ 15905, USA \\ *Corresponding author: muzammilkhan972@yahoo.com
}

\begin{abstract}
Sodium-glucose co-transporter-2 (SGLT-2) inhibitor is the latest class of anti diabetic medication that improves glycemic control in insulin independent fashion by increasing urinary loss of filtered glucose. Since its introduction in 2013, several cases of euglycemic DKA have been reported in patients being treated with SGLT-2 inhibitors. Blood glucose levels in range lower than expected for DKA makes the diagnosis challenging if clinical suspicion for euglycemic DKA is not high. We report a case of a patient being treated with canagliflozin who presented with DKA, AKI and mild hyperglycemia that was complicated by stress-induced cardiomyopathy.
\end{abstract}

Keywords: sodium-glucose co-transporter-2 (SGLT-2) inhibitor, euglycemic DKA

Cite This Article: Muzammil Khan, Shaza Khalid, Asghar Marwat, and Hassan Mehmood, "A Case of Euglycemic Diabetic Ketoacidosis due to Canagliflozin Complicated by Takotsubo Cardiomyopathy." American Journal of Medical Case Reports, vol. 6, no. 1 (2018): 1-3. doi: 10.12691/ajmcr-6-1-1.

\section{Introduction}

In March 2013, first Sodium-glucose co-transporter-2 (SGLT-2) inhibitor, canagliflozin, was approved for the treatment of type 2 diabetes mellitus by the US food and drug agency (FDA) (1). SGLT-2 inhibitors reversibly inhibit the SGLT-2 located in the proximal convoluted tubule of the kidney where $90 \%$ of filtered sodium and glucose is reabsorbed. By increasing urinary loss of glucose, SGLT-2 improves glycemic control [3]. Major adverse effect of SGLT-2 inhibitors is to increase the propensity towards ketoacidosis with lower than anticipated blood glucose level although the incidence of DKA has not found to be greater than the general diabetes population [6]. It has been proposed that acute illness, starvation, deficiency of insulin or oral secretagouges increases the release of counter regulatory hormones that promotes gluconeogenesis and ketogenesis [2,11]. SGLT-2 inhibition is reversed quickly after cessation of SGLT-2 inhibitors.

\section{Case Report}

A 56-year-old woman was bought to the emergency room after she was found confused by her husband when she woke up in the morning. She had 15-year history of type 2 diabetes. She was managed with metformin, exenatide and glipizide. Canagliflozin $300 \mathrm{mg}$ daily was started 10 days prior to her presentation due to an elevated $\mathrm{HbA} 1 \mathrm{C}$ of $11.9 \%$. Her physical examination revealed tachypnea (respiratory rate $24 /$ minute), tachycardia (heart rate $110 / \mathrm{min}$ ), temperature 36.5 degree Celsius and altered mental status; oriented to person only. Labs were notable for BUN $13 \mathrm{mg} / \mathrm{dl}$, creatinine of $1.4 \mathrm{mg} / \mathrm{dl}$ compared with $(\mathrm{C} / \mathrm{W}) 0.7 \mathrm{mg} / \mathrm{dl}$ at baseline before canagliflozin was started, bicarbonate $<5 \mathrm{mEq} / \mathrm{L}$, anion gap of 28 , beta hydroxybutyrate of $11.81 \mathrm{mmol} / \mathrm{L}$, glucose modestly elevated to $170 \mathrm{mg} / \mathrm{dl}$, arterial pCO2 of $24 \mathrm{~mm} \mathrm{Hg}$ and arterial $\mathrm{pH}$ of 6.94 . Urinalysis was pertinent for glucose $>$

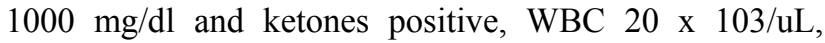
lactic acid $2.7 \mathrm{mmol} / \mathrm{L}$. Troponin I was $0.05 \mathrm{ng} / \mathrm{ml}, \mathrm{EKG}$ revealed wide complex tachycardia that appeared to be sinus tachycardia and left bundle branch block that was old c/w previous EKG.

CT head without contrast did not show any acute intracranial pathology, chest x-ray did show left lower lobe consolidation.

The patient was transferred to ICU, started on dextrose containing IV fluids, insulin infusion and appropriate antibiotics for health care associated pneumonia. To protect her airway as her sensorium was altered, she was intubated and started on mechanical ventilation. While central venous access (CVA) was being established, she went into ventricular tachycardia (VT) and then became pulseless. Return of spontaneous circulation (ROSC) was achieved after 4 minutes of resuscitation per ACLS guidelines. It was felt that CVA induced the VT, as she did not had any further VT.

On further evaluation after ROSC, EKG revealed sinus tachycardia and left bundle branch block with no new changes c/w previous EKG, no electrolyte abnormalities, severe acidosis with bicarbonate level $6 \mathrm{mEq} / \mathrm{L}$, troponin elevated to $0.74 \mathrm{ng} / \mathrm{ml}$ and pleatued at $0.63 \mathrm{ng} / \mathrm{ml} \mathrm{c} / \mathrm{w} 0.05$ 
$\mathrm{ng} / \mathrm{ml}$ at admission with normal $\mathrm{CK}$ and CK-MB. Transthoracic echocardiography (TTE) obtained 12 hours after the cardiac arrest revealed severely decreased left ventricular (LV) systolic function, $\mathrm{LV}$ ejection fraction (EF) $21 \% \mathrm{c} / \mathrm{w} 61 \%$ on prior TTE 6 months ago, normal LV size and LV wall thickness, and septal wall motion abnormality.

She received 5 liters of intravenous (IV) fluids. Insulin drip was bridged to subcutaneous insulin after normalization of her anion gap and serum bicarbonate level, IV fluids were stopped as her creatinine trended down to normal and IV antibiotics were switched to oral antibiotics after extubation.

Her LV dysfunction was felt to be due to stress induced cardiomyopathy as her LV EF improved to $55 \%$ on TTE a week later, her troponin pleatued at $0.63 \mathrm{ng} / \mathrm{ml}$ and she did not had new electrocardiographic ischemic changes. She refused to undergo cardiac catheterization.

She was discharged on basal and bolus weight based subcutaneous insulin regimen and her oral diabetic medications were held.

\section{Discussion}

SGLT 2 inhibitor is the newest class of anti diabetic medications approved recently by the US food and drug agency to be used in conjunction with diet and exercise for treatment of type 2 diabetes mellitus [1]. Canagliflozin, dapagliflozin and empaglifilozin are the SGLT-2 inhibitors currently approved by the FDA [2]. Canagliflozin decreases the renal threshold for glucose excretion by reversibly blocking SGLT 2 located in the proximal convoluted tubule of the kidney and thus increases loss of glucose in the urine thereby decreasing serum glucose concentration [3].

Canagliflozin is orally administered; it reaches its steady state in 4 days with minimal accumulation after further redosing. SGLT 2 inhibition should disappear 2 to 3 days after discontinuation of canagliflozin as its half-life is dose dependent, 10.6 and 13.1 hours for $100 \mathrm{mg}$ and $300 \mathrm{mg}$ of canagliflozin respectively [4]. Along with favorable effects on blood pressure and body weight, Canagliflozin causes sustained reduction of $\mathrm{HbA} 1 \mathrm{C}$ [5].

The most common adverse reactions associated with canagliflozin are genital mycotic infections, urinary tract infections, osmotic diuresis, and reduced intravascular volume [1] but FDA revised the labels more recently to include warnings and precautions about the risks of ketoacidosis with SGLT 2 inhibitors [6].

The incidence of DKA was reported to be $0.52,0.76$, and 0.24 per 1000 patient-years for canagliflozin $100 \mathrm{mg}$, $300 \mathrm{mg}$, and a non-canagliflozin comparator, respectively by a meta-analysis of canagliflozin trials. According to several studies, the overall frequency of reported events suggestive of DKA is less than $0.1 \%$ [7]. Search of the FDA Adverse Event Reporting System (FAERS) database identified 73 cases of ketoacidosis in patients with type 1 or type 2 diabetes treated with SGLT 2 inhibitors from March 2013 to May 2015 [8]. DKA associated with SGLT 2 inhibitors usually does not have elevated blood glucose level due to persistent excretion of glucose in urine [4].
Euglycemic DKA is defined by anion gap metabolic acidosis $(\mathrm{PH}<7.3$ and serum bicarbonate $<18 \mathrm{mEq} / \mathrm{L}$ ), presence of ketones in blood or urine and blood glucose $<200 \mathrm{mg} / \mathrm{dl}$ [9]. The fall in the blood glucose level as glucose is lost in the urine decreases the insulin level in the body and increases glucagon level thus favoring gluconeogenesis and ketogenesis [10]. Decrease in the Insulin or secretagouge dose, starvation and acute illness have been identified as risk factors for euglycemic DKA as these conditions increase the counter regulatory hormones including cortisol, glucagon and epinephrine which promotes ketogenesis [2,11]. Given reduced oral intake and concurrent HCAP, acute illness and starvation might have played a role in activating the ketosis pathway in our patient.

Stress cardiomyopathy is a syndrome characterized by transient regional left ventricular systolic dysfunction that mimics myocardial infarction, but in the absence of angiographic evidence of obstructive coronary artery disease. Stress cardiomyopathy is treated with supportive care [12].

\section{Conclusion}

1. Canagliflozin is associated with severe anion gap metabolic acidosis with ketoacidosis and normal to modestly elevated glucose level.

2. Close follow up is required to monitor basic serum chemistry for evidence of increasing anion gap acidosis after starting canagliflozin

3. Even with normal or modestly elevated blood glucose level, these patients should be treated similar to DKA

4. SGLT-2 inhibitors should be avoided when poor oral intake is anticipated

\section{Statement of Competing Interests}

The author(s) declare(s) that there is no conflict of interest regarding the publication of this paper

\section{References}

[1] Gelaye A, Haidar A, Kassab C, Kazmi S, Sinha P. Severe ketoacidosis associated with canagliflozin (Invokana): a safety concern. Case reports in critical care. 2016 Mar 21;2016.

[2] Candelario N, Wykretowicz J. The DKA that wasn't: a case of euglycemic diabetic ketoacidosis due to empagliflozin. Oxford medical case reports. 2016 Jul 1;2016(7):144-6.

[3] Devineni D, Polidori D. Clinical pharmacokinetic, pharmacodynamic, and drug-drug interaction profile of canagliflozin, a sodium-glucose co-transporter 2 inhibitor. Clinical pharmacokinetics. 2015 Oct 1;54(10):1027-41.

[4] Kelmenson DA, Burr K, Azhar Y, Reynolds P, Baker CA, Rasouli N. Euglycemic Diabetic Ketoacidosis With Prolonged Glucosuria Associated With the Sodium-Glucose Cotransporter-2 Canagliflozin. Journal of Investigative Medicine High Impact Case Reports. 2017 Jun;5(2):2324709617712736.

[5] Rosenthal N, Meininger G, Ways K, Polidori D, Desai M, Qiu R, Alba M, Vercruysse F, Balis D, Shaw W, Edwards R. Canagliflozin: a sodium glucose co - transporter 2 inhibitor for the treatment of type 2 diabetes mellitus. Annals of the New York Academy of Sciences. 2015 Nov 1;1358(1):28-43. 
[6] US Food and Drug Administration. SGLT2 inhibitors: drug safety communication-labels to include warnings about too much acid in the blood and serious urinary tract infections [Internet]. FDA Drug Safety Communication. 2015 Dec.

[7] Erondu N, Desai M, Ways K, Meininger G. Diabetic ketoacidosis and related events in the canagliflozin type 2 diabetes clinical program. Diabetes Care. 2015 Jul 22:dc151251.

[8] US Food and Drug Administration. FDA Drug Safety Communication: FDA warns that SGLT2 inhibitors for diabetes may result in a serious condition of too much acid in the blood. Bethesda, MD: US Food and Drug Administration, 2015 May 15. 2015 May 15.
[9] Modi A, Agrawal A, Morgan F. Euglycemic Diabetic Ketoacidosis: A Review. Current Diabetes Reviews. 2017 Jun 1;13(3):315-21.

[10] Ogawa W, Sakaguchi K. Euglycemic diabetic ketoacidosis induced by SGLT2 inhibitors: possible mechanism and contributing factors. Journal of diabetes investigation. 2016 Mar $1 ; 7(2): 135-8$.

[11] Clement M, Senior P. Euglycemic diabetic ketoacidosis with canagliflozin. Canadian Family Physician. 2016 Sep 1;62(9):725-8.

[12] Sato H, Tateishi H, Uchida T. Takotsubo-type cardiomyopathy due to multivessel spasm. Clinical aspect of myocardial injury: from ischemia to heart failure. 1990;56. 